OPEN ACCESS

Edited by:

Jun Lu,

Auckland University of Technology,

New Zealand

Reviewed by: Jagadish Hiremath,

National Institute of Veterinary Epidemiology And Disease Informatics

(ICAR), India

Hiroyuki Wakiguchi,

Yamaguchi University, Japan

lan Christopher Davis,

The Ohio State University,

United States

*Correspondence:

Xunlong Shi

xunlongshi@fudan.edu.cn

Yiou Cao

yioucao_doctor@126.com

${ }^{\dagger}$ These authors have contributed equally to this work

Specialty section:

This article was submitted to Experimental Pharmacology and Drug Discovery,

a section of the journal

Frontiers in Pharmacology

Received: 22 April 2020 Accepted: 05 August 2020 Published: 06 October 2020

Citation:

Geng P, Zhu H, Zhou W, Su C, Chen $M$, Huang $C$, Xia $C$, Huang $H$, Cao Y and Shi X (2020) Baicalin Inhibits Influenza A Virus Infection via Promotion of M1 Macrophage Polarization.

Front. Pharmacol. 11:01298. doi: 10.3389/fphar.2020.01298

\section{Baicalin Inhibits Influenza A Virus Infection via Promotion of M1 Macrophage Polarization}

\author{
Ping Geng ${ }^{1 \dagger}$, Haiyan Zhu ${ }^{1 \dagger}$, Wei Zhou ${ }^{2}$, Chang Su ${ }^{3}$, Mingcang Chen ${ }^{4}$, \\ Chenggang Huang ${ }^{4}$, Chengjie Xia ${ }^{1}$, Hai Huang ${ }^{1}$, Yiou $\mathrm{Cao}^{3 *}$ and Xunlong Shi ${ }^{1 *}$
}

1 Department of Biological Medicines \& Shanghai Engineering Research Center of Immunotherapeutics, Fudan University School of Pharmacy, Shanghai, China, 2 Department of Chemistry, Fudan University, Shanghai, China, ${ }^{3}$ Department of Surgery, Minhang Hospital, Fudan University, Shanghai, China, ${ }^{4}$ Shanghai Institutes of Materia Medica, Chinese Academy of Sciences, Shanghai, China

Background and Aims: The natural compound baicalin (BA) possesses potent antiviral properties against the influenza virus. However, the underlying molecular mechanisms of this antiviral activity and whether macrophages are involved remain unclear. In this study, we, therefore, investigated the effect of BA on macrophages.

Methods: We studied macrophage recruitment, functional phenotypes (M1/M2), and the cellular metabolism via flow cytometry, qRT-PCR, immunofluorescence, a cell culture transwell system, and GC-MS-based metabolomics both in vivo in $\mathrm{H} 1 \mathrm{~N} 1 \mathrm{~A}$ virus-infected mice and in vitro.

Results: BA treatment drastically reduced macrophage recruitment $\left(\mathrm{CD} 11 \mathrm{~b}^{+}, \mathrm{F} 4 / 80^{+}\right)$by approximately $90 \%$ while maintaining the proportion of M1-polarized macrophages in the bronchoalveolar lavage fluid of infected mice. This BA-stimulated macrophage M1 phenotype shift was further verified in vitro in ANA-1 and primary peritoneal macrophages by measuring macrophage M1 polarization signals (CD86, iNOS, TNF- $\alpha$, iNOS/Arg-1 ratio, and IL-1 $\beta$ cleavage). Meanwhile, we observed an activation of the IFN pathway (upregulation of IFN- $\beta$ and IRF-3), an inhibition of influenza virus replication (as measured by the $M$ gene), and distinct cellular metabolic responses in BA-treated cells.

Conclusion: BA triggered macrophage M1 polarization, IFN activation, and other cellular reactions, which are beneficial for inhibition of $\mathrm{H} 1 \mathrm{~N} 1 \mathrm{~A}$ virus infection.

Keywords: macrophage, M1 polarization, influenza virus, baicalin, metabolism

\section{INTRODUCTION}

Influenza viruses are the most frequent human pathogens and are responsible for many complications, such as viral pneumonia (Rello, 2017; http://www.who.int/influenza/surveillance_ monitoring/updates/latest_update_GIP_surveillance/en/). Novel mutant influenza A viruses, such as avian $\mathrm{H7N}$ 9, do not respond well to current antiviral therapies and can cause substantial excess mortality during pandemics and the annual flu season (Zhou et al., 2017). Thus, the development of novel therapeutic strategies is an urgent matter. 
Macrophages are one of the primary sources of inflammatory cytokines (such as TNF- $\alpha /$ chemokines) and act as critical modulators of influenza virus infection severity and the development of lethal pulmonary injury (Cardani et al., 2017). Their phenotype-classically activated (M1) or alternatively activated (M2) - is recognized as a key regulating factor in the initiation, progression, and termination of numerous inflammatory diseases (Li et al., 2018), in particular in influenza diseases (Arora et al., 2018). Therefore, modulating macrophage polarization might represent a possible therapeutic strategy for influenza virus infection (Vergadi et al., 2017; Halstead et al., 2018).

Chemicals with anti-influenza activity derived from natural products are rapidly emerging, some of which may constitute possible novel drug options (Cardani et al., 2017). Baicalin (BA) is a natural flavonoid present in several medicinal plants and is shown to prevent death, inhibit lung consolidation, and reduce viral replication (Ding et al., 2014; Nayak et al., 2014; da Silva et al., 2017). In a previous study, we found that BA could inhibit influenza virus-induced autophagy in macrophages (Zhu et al., 2015). However, it is still unknown whether BA exerts its antiviral effects via modulation of macrophage functions.

Reprogramming of the host cellular metabolism triggered by the influenza virus is highly associated with inflammation and immune cell activation (Chandler et al., 2016), and metabolomics is believed to be a highly sensitive and specific tool for the prognosis of viral pneumonia in anti-influenza clinical therapies (Tisoncik-Go et al., 2016). In this research, we use metabolomics (GC-MS analysis) to evaluate the effects of BA on macrophages.

\section{MATERIALS AND METHODS}

\section{Chemicals and Reagents}

BA (98\% HPLC purity; ChromaBio, Chengdu, China) was dissolved in DMSO to generate a $50-\mathrm{mM}$ stock solution and stored at $4^{\circ} \mathrm{C}$. It was subsequently diluted in cell culture medium to different working solutions. For animal experiments, BA and ribavirin solutions were prepared with 0.5\% CMC-Na.

Antibodies against mouse CD11b, F4/80, CD86, CD206, and cleaved interleukin-1 $\beta$ (IL-1 $\beta$ ) were obtained from Biolegend (San Diego, CA, USA). Secondary antirabbit IgG Fab2 Alexa Fluor ${ }^{\circledR} 488$ molecular probe was purchased from Santa Cruz Biotechnology (Santa Cruz, CA, USA). All other chemicals were obtained from Beyotime Biotechnology (Shanghai, China).

\section{METHODS}

\section{Experimental H1N1 Infection}

Male BALB/c mice (16-18 g) were purchased from the Shanghai SLACCAS Laboratory Animal Co., Ltd. (Shanghai, China). Mice were housed under specific pathogen-free (SPF) conditions and given free access to sterile water and standard mouse chow. All experimental protocols were approved by the Animal Experiment Committee of Fudan University (Shanghai, China) (Approval number 2018-03-WY-SXL-01).
The influenza virus A/FM/1/47 (H1N1) was supplied by the Shanghai Center for Disease Control \& Prevention (Shanghai, China) and was stored in aliquots at $-70^{\circ} \mathrm{C}$. For each experiment, an aliquot was thawed to ensure the use of a fresh preparation.

Mice were randomly divided into four groups with 8 mice per group: control mice (Normal group), virus-infected mice (Virus group), BA-treated infected mice (BA group), and ribavirintreated infected mice (Ribavirin group). All mice except control mice were anesthetized under $1 \%$ pentobarbital and infected intranasally (i.n.) with $5 \times \mathrm{LD}_{50}$ of $\mathrm{A} / \mathrm{FM} / 1 / 47$ (H1N1). Two hours postinfection, mice were treated with $80 \mathrm{mg} / \mathrm{kg}$ of BA (BA group) (Ding et al., 2014), $20 \mathrm{mg} / \mathrm{kg}$ of ribavirin (Ribavirin group) (Ridnour et al., 2015), and 0.5\% CMC-Na (Normal and Virus groups) by gavage once daily for 7 days. The experiments were repeated twice, and all mice were continuously monitored for survival and body weight loss.

\section{Experimental H1N1 Infection in Macrophages and Epithelial Cells}

For in vitro experiments, the $\mathrm{H} 1 \mathrm{~N} 1 / \mathrm{PR} / 8 / 34 \mathrm{TC}$ adapted strain was obtained from ATCC (VR,1469 AC) and stored in aliquots at $-70^{\circ} \mathrm{C}$. For each experiment, an aliquot was thawed to ensure the use of a fresh preparation.

Murine ANA-1 macrophages and epithelial BEAS-2B cells were obtained from the Cell Bank of the Shanghai Institute of Biochemistry and Cell Biology of the Chinese Academy of Sciences (Shanghai, China).

Murine ANA-1 macrophages (Fabozzi et al., 2018) and primary peritoneal macrophages (isolated from SPF Balb/C mice) were cultured in RPMI 1640 medium $\left(1 \times 10^{5}\right.$ cells $\left./ \mathrm{ml}\right)$ with $10 \%(\mathrm{v} / \mathrm{v})$ heat-inactivated fetal bovine serum (Gibco), $0.3 \mathrm{mg} / \mathrm{mL}$ l-glutamine, $100 \mathrm{U} / \mathrm{mL}$ penicillin, and $100 \mu \mathrm{g} / \mathrm{mL}$ streptomycin at $37^{\circ} \mathrm{C}$ under $5 \% \mathrm{CO}_{2}$.

BEAS-2B cells (Weinhofer et al., 2018) were cultured in the DMEM supplemented with $10 \%(\mathrm{v} / \mathrm{v})$ heat-inactivated fetal bovine serum (Gibco), $0.3 \mathrm{mg} / \mathrm{mL}$ l-glutamine, $100 \mathrm{U} / \mathrm{mL}$ penicillin, and $100 \mu \mathrm{g} / \mathrm{mL}$ streptomycin at $37^{\circ} \mathrm{C}$ under $5 \% \mathrm{CO}_{2}$.

The cells were infected with $10 \times$ the median tissue culture infectious dose $\left(10 \times \mathrm{TCID}_{50}\right)$ of $\mathrm{H} 1 \mathrm{~N} 1$ virus $(\mathrm{PR} / 8 / 34, \mathrm{ATCC})$ for $2 \mathrm{~h}$ and then transferred to BA solution (diluted in 1640 medium for macrophages and DMEM for BEAS-2B cells) for 24 or $48 \mathrm{~h}$.

\section{The Phenotype of Macrophages In Vivo and In Vitro}

For the in vivo study, the bronchoalveolar lavage fluid (BALF) of different grouped mice was collected three times by lavage with $0.5 \mathrm{~mL}$ ice-cold PBS under pentobarbital anesthesia at 4 days postinfection. The collected BALF was centrifuged at $700 \times g$ at $4^{\circ}$ $\mathrm{C}$ for $5 \mathrm{~min}$, and the harvested cells were resuspended in $200 \mu \mathrm{L}$ PBS. The cells were stained with fluorescently labeled antibodies against the following mouse proteins: $\mathrm{CD} 11 \mathrm{~b}^{+}, \mathrm{F} 480^{+}$ (macrophages), CD11b ${ }^{+}, \mathrm{F} 4 / 80^{+}, \mathrm{CD}^{+} 6^{+}$(M1 phenotype macrophage), and $\mathrm{CD}_{11 \mathrm{~b}}{ }^{+}, \mathrm{F} 4 / 80^{+}, \mathrm{CD}_{20}{ }^{+}$(M2 phenotype macrophage) (Schmittgen and Livak, 2008; Murray et al., 2014).

For the in vitro study (24 and $48 \mathrm{~h}$ postinfection), infected ANA-1 macrophages were stained with fluorescently labeled 
antibodies against the following mouse proteins: $\mathrm{CD}^{+} 6^{+}(\mathrm{M} 1$ phenotype) and CD206 ${ }^{+}$(M2 phenotype).

Flow cytometry analysis was performed on a BD FACS Calibur (BD Biosciences, USA). All plots shown on populations were gated on lymphocytes by forward and side scattering, collecting 100,000 cells per sample. Compensation settings were adjusted using isotype controls. Data analysis was performed using the FlowJo software (Ashland, OR, USA).

High levels of cellular iNOS and TNF- $\alpha$ are often observed in M1 polarized macrophages. Thus, we quantified cellular iNOS (using the Diaminofluorescein-FM diacetate probe; Nitric Oxide Synthase Assay Kit, S0025, Beyotime, Shanghai, China) and TNF- $\alpha$ (using a TNF- $\alpha$ ELISA kit) to evaluate the macrophage polarization.

\section{Real-Time Quantitative RT-PCR (qRT- PCR) Analysis}

For cell experiments at 24 and $48 \mathrm{~h}$ postinfection, total RNA was isolated using TRIzol reagent (Invitrogen, CA, USA) according to the manufacturer's instructions. The RNA was converted into cDNA using a Reverse Transcription Kit (Takara, Japan). qRTPCR was performed using the StepOne Plus RT-PCR System (Applied Biosystems) using the following thermocycling parameters: $94^{\circ} \mathrm{C}$ for $5 \mathrm{~min}$, followed by 40 cycles of $94^{\circ} \mathrm{C}$ for $5 \mathrm{~s}$ and $60^{\circ} \mathrm{C}$ for $30 \mathrm{~s}$. The mRNA levels of iNOS, Arg-1, influenza $M$ gene, INF- $\beta, I L-10, I R F-3$, and IRF-7 were normalized to the geometric mean of GAPDH mRNA levels by using a $\triangle \triangle C T$ method (Lin et al., 2010). The primers used in this study are shown in Table 1.

\section{Macrophage Recruitment Assay (Transwell System)}

ANA-1 macrophages (labeled with GFP lentiviral vectors from Genechem, Shanghai, China) were cultivated in the upper chamber of 24 -well Transwell plates $\left(10^{4}\right.$ cells/well). ANA-1 or BEAS-2B cells were grown in the lower room $\left(10^{5}\right.$ cells $\left./ \mathrm{mL}\right)$. The cells in lower chambers were infected with the influenza $\mathrm{PR} / 8 / 34$

TABLE 1 | Primers for qPCR.

\begin{tabular}{lll}
\hline Primer name & & Sequence (5'to3') \\
\hline Influenza VirusM gene & Forward & GACCGATCCTGTCACCTCTGAC \\
& Reverse & AGGGCAT CTGGACAAAGCGTCTA \\
iNOS & Forward & TCCTGGAGGAAGTGGGCCGAAG \\
& Reverse & CCTCCACGGGCCCGGTACTC \\
Arg-1 & Forward & CAGAAGAATGGAAGAGTCAG \\
& Reverse & CAGATATGCAGGGAGTCAC \\
IRF3 & Forward & TCCGCTAGTCTACAGCCCT \\
& Reverse & ACACTGTTACCTGATCTGCCC \\
IRF7 & Forward & CAGCTCAGCAGCCTACCAC \\
& Reverse & TGACATTGGCGCTGTGAAGAG \\
IL-1 $\beta$ & Forward & TGCCACCTITGACAGTGATG \\
& Reverse & TGATGTGCTGCTGCGAGATT \\
IL-10 & Forward & GGACAACATACTGCTAACCGAC \\
IFN- $\beta$ & Reverse & TGGATCATTCCGATAAGGCTTG \\
& Forward & TGCATCTTCTCCGTCATCTC \\
GAPDH & Reverse & TAGCAGCCGACACCAGCCTG \\
& Forward & ACAGCCTCAAGATCATCAGCA \\
& Reverse & ATGAGTCCTTCCACGATACCA
\end{tabular}

virus $\left(10 \times \mathrm{TCID}_{50}\right)$ for $2 \mathrm{~h}$ and then transferred to fresh culture medium or BA dilutions.

Macrophage recruitment was observed under a Leica EL6000 microscope (Leica Microsystems CMS GmbH).

\section{Cell Immune-Fluorescence Staining Analysis}

Cells were fixed with $4 \%$ paraformaldehyde buffer for $30 \mathrm{~min}$, permeabilized with $0.5 \%$ Triton X-100 for $20 \mathrm{~min}$, and blocked with QuickBlock $^{\mathrm{TM}}$ Blocking Buffer for $30 \mathrm{~min}$ at room temperature. The cells were probed with IL-1 $\beta$ antibodies (1:1000 dilution) at $4^{\circ} \mathrm{C}$ overnight and subsequently detected with antirabbit IgG Fab2 Alexa Fluor ${ }^{\circledR} 488$ molecular probes (1:2000 dilution). After counterstaining cell nuclei with DAPI (1:1000 dilution of $100 \mu \mathrm{g} / \mathrm{mL}$ stock solution) for at least $3 \mathrm{~min}$, immunofluorescent images were obtained using a Leica EL6000 microscope (Leica Microsystems).

\section{Metabolomics}

The cell samples were frozen and thawed at room temperature. Three hundred $\mu \mathrm{L}$ methanol were added to $100 \mu \mathrm{L}$ of the homogenate to precipitate the protein, and the mixture was shaken vigorously for $30 \mathrm{~s}$. Ribitol $(10 \mu \mathrm{L}$ at $0.02 \mathrm{mg} / \mathrm{mL})$ was added to the sample as the internal standard. The mixture was then ultrasonically extracted for $10 \mathrm{~min}$, followed by centrifugation $(3000 \times \mathrm{g})$ for another $10 \mathrm{~min}$. Then, $150 \mu \mathrm{L}$ of the supernatant was transferred to the GC vial and evaporated to dryness under a stream of nitrogen gas. The chemical derivatization of the cell metabolites was carried out using the combination of methoxymation and silylation. A $30-\mu \mathrm{L}$ aliquot of methoxyamine pyridine solution $(20 \mathrm{mg} / \mathrm{mL})$ was added to the vial. The methoxymation was performed at $37^{\circ} \mathrm{C}$ for $90 \mathrm{~min}$. Then, $30 \mu \mathrm{L}$ of bis-(trimethylsilyl)-trifluoroacetamide plus $1 \%$ trimethylchlorosilane were added to each sample. The samples were mixed on a vortex for $1 \mathrm{~min}$ and incubated at $70^{\circ} \mathrm{C}$ for $60 \mathrm{~min}$. After incubation, samples were again vortexed for $1 \mathrm{~min}$ and transferred to vials for GC/MS analysis (Agilent 7890A GC system).

The GC/MSD Chem Station Software (Agilent, Shanghai, China) was used for the autoacquisition of GC total ion chromatograms and fragmentation patterns. For each peak, the software generates a list of similarities comparing with every substance within the National Institute of Standards and Technology (NIST) mass spectra library. Peaks with a similarity index of more than $70 \%$ were assigned compound names, and those having less than $70 \%$ similarity were listed as unknown metabolites. Each sample was characterized by the same number of variables, and each of these variables was represented across all observations with the same sequence. Thus, a data matrix was generated by the intensities of the commensal peaks from all samples to characterize the biochemical pattern of each sample (Wang et al., 2016).

To create the metabolite heat map, we normalized data for each metabolite [(original value-mean)/standard deviation], obtaining a $z$-score, and then set the minimum value to -2 (dark blue), the median to 0 (white), and maximum value to 2 (dark red). 


\section{Statistical Analysis}

All statistical analyses were performed using GraphPad Prism for Windows (Version 6.0). The Gehan-Breslow-Wilcoxon test was used to analyze the survival of mice, and other experimental data were evaluated by using the Student's $t$-test (comparison of two groups) or one-way ANOVA (for comparisons with more than two groups sample). In all cases, probability levels less than 0.05 $(P<0.05)$ were considered to indicate statistical significance.

\section{RESULTS}

\section{BA Significantly Inhibits the Recruitment of Macrophages Into the Lung Tissues of H1N1-Infected Mice}

As shown in Figure 1A, the infection with $5 \times \mathrm{LD}_{50}$ influenza virus A/FM/1/47 caused $75 \%$ mortality within 14 days postinfection, accompanied by visible body weight loss. Treatment with 80 $\mathrm{mg} / \mathrm{kg}$ BA attenuated death and body weight loss of infected mice (Figure 1B).

We collected the BALF of infected mice (control, virus, BAtreated) at 4 days postinfection and analyzed macrophage recruitment and phenotype. As shown in Figure 1C, very few macrophages were present in lung tissues of normal mice although H1N1 infection caused the massive recruitment of macrophages (nearly $10 \% \mathrm{CD} 1 \mathrm{~b}^{+}-\mathrm{F} 4 / 80^{+}$macrophages per $10^{5}$ cells) to the lungs. BA treatment $(80 \mathrm{mg} / \mathrm{kg})$ significantly reduced virus- induced macrophage recruitment ( $1 \%$ of $10^{5}$ cells). Furthermore, we then analyzed the polarization of recruited macrophages from the virus and BA groups and found that BA treatment resulted in a higher proportion of M1 macrophages than in virus mice (97\%

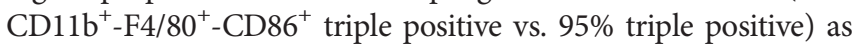
shown in Figure 1D.

We, therefore, concluded that recruitment and polarization of macrophages might play a critical role in the antiviral effects of BA.

\section{BA triggers macrophage M1 polarization and activates IFN signaling pathway}

ANA-1 macrophages are a commonly used murine macrophage cell line (Evavold et al., 2018), which we previously used to replicate influenza virus infection in vitro (Zhu et al., 2015). Therefore, we used ANA-1 cells to evaluate macrophage recruitment and polarization after $\mathrm{H} 1 \mathrm{~N} 1 \mathrm{PR} / 8 / 34$ infection (10 $\mathrm{TCID}_{50}$ ) with or without BA treatment. As shown in Figure 2A regardless of whether cells were healthy or infected, BA treatment ( 1 and $10 \mu \mathrm{M}$ ) promoted an M1 polarization of macrophages by enhancing CD86 positive cells, but had a limited effect on other macrophages (such as CD86-CD206 double-positive, CD86CD206 double-negative, and CD206 single positive macrophages).

In Figure 2B, we show that BA treatment $(10 \mu \mathrm{M})$ significantly enhanced cellular iNOS and TNF- $\alpha$ levels in infected ANA-1 cells at $24 \mathrm{~h}$ postinfection but decreased iNOS levels at $48 \mathrm{~h}$ postinfection.
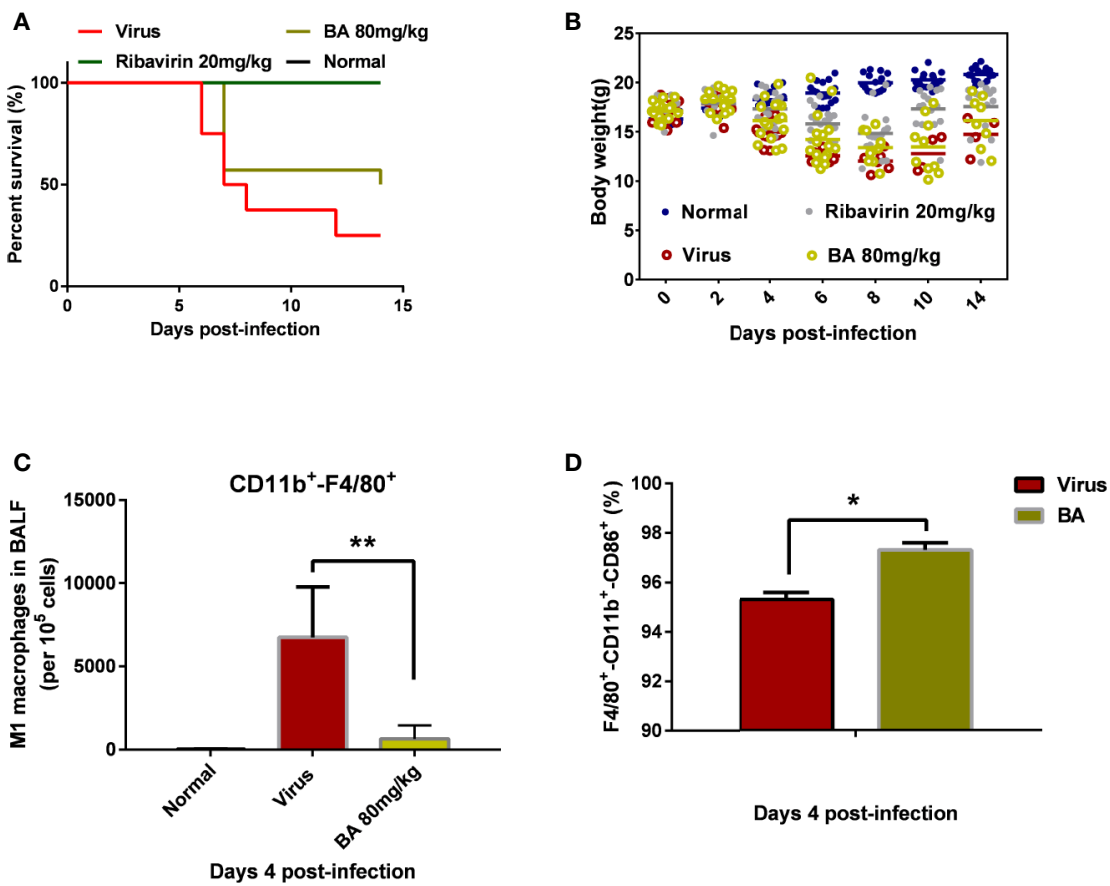

FIGURE 1 | BA reduces the mortality of infected mice and suppresses the recruitment of macrophages in BALF. BALB/c mice were i.n. infected with influenza virus A H1N1 FM/1/47 strain at $5 \times L_{50}$ and received BA treatment (80 mg/kg/day) for 7 days. (A) Survival curve. (B) Body weight curve. (C-D) Macrophage phenotype in the BALF (Normal, Virus, and BA treatment, 4 days postinfection). Data are presented as mean \pm SD. ${ }^{*} p \leq 0.05,{ }^{* *} p \leq 0.01$ compared with virus samples. 
A

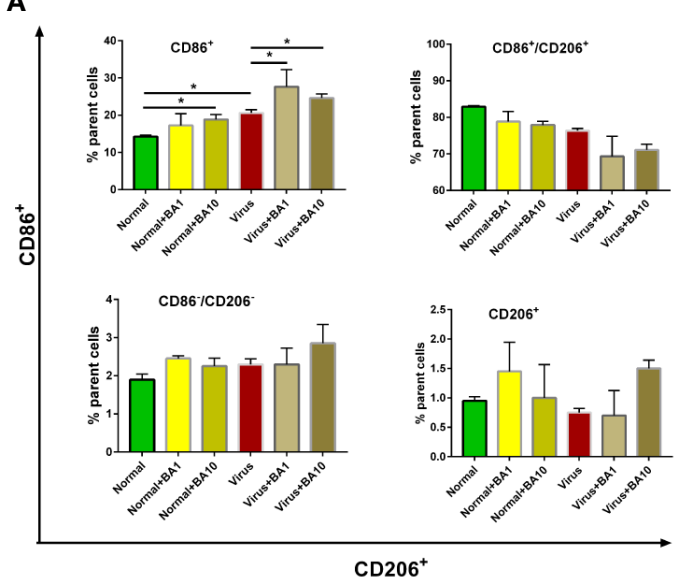

C
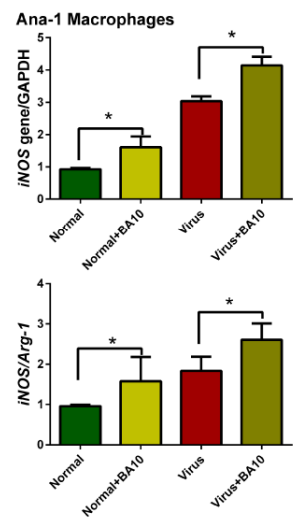

B
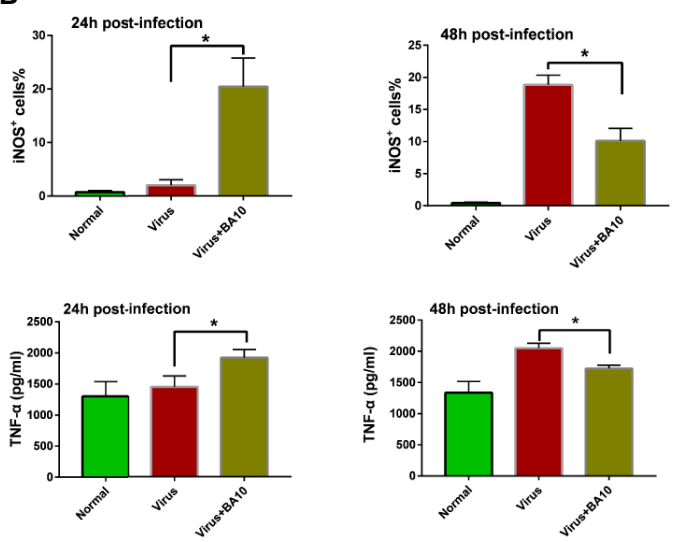

D
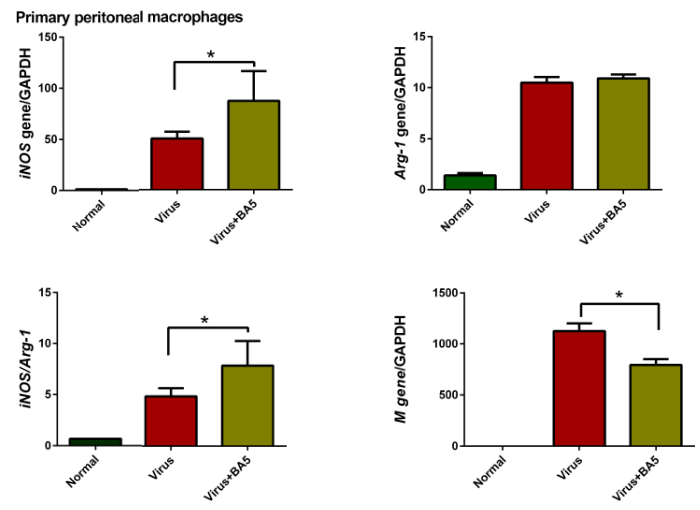

\section{E}
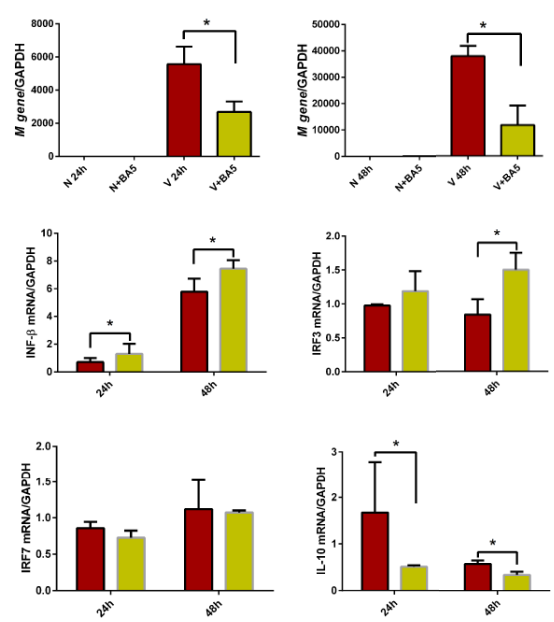

FIGURE 2 | triggers macrophage M1 polarization and activates the IFN signaling pathway. Macrophages (ANA-1 cells and primary peritoneal macrophages, $2 \times 10^{5}$ cells/ well) were infected with influenza virus $\mathrm{H} 1 \mathrm{~N} 1 \mathrm{PR} / 8 / 34$ strain $\left(10 \times \mathrm{TCID}_{50}\right)$ and cultured in 6 -well plates. The medium was replaced by BA dilutions (1 and $\left.10 \mu \mathrm{M}\right)$ after $2 \mathrm{~h}$ of incubation. Uninfected cells were used as the standard control. (A) Macrophage polarization of ANA-1 cells. At $24 \mathrm{~h}$ postinfection, ANA-1 cells were stained with fluorescently labeled antibodies against the following mouse proteins: $\mathrm{CD}^{+} 6^{+}$(M1 phenotype) and $\mathrm{CD}^{206^{+}}$(M2 phenotype). The population of labeled cells was analyzed by flow cytometry. (B) At 24 and 48 h postinfection, cellular iNOS and secreted TNF- $\alpha$ were further quantified by Nitric Oxide Synthase Assay Kit and ELISA Kit. (C, D) At $24 \mathrm{~h}$ postinfection, total RNA of cell samples (10 $\mu \mathrm{M}$ BA treated-ANA-1 cells; $5 \mu \mathrm{M}$ BA treated-primary peritoneal macrophages) was isolated and reverse-transcribed to CDNA for analysis of macrophage phenotype (iNOS and Arg-1 gene) and influenza virus replication ( $M$ gene). (E) Comparative analysis of IFN, IRFs, and other related gene transcription in ANA-1 cell samples (24 and $48 \mathrm{~h}$ postinfection). Data are presented as mean $\pm \mathrm{SD} .{ }^{*} p \leq 0.05,{ }^{\star \star} p \leq 0.01$ compared with virus samples. 
We further analyzed macrophage M1 phenotype-related gene expression ( $i N O S$ and Arg-1) in ANA-1 macrophages and primary peritoneal macrophages. In ANA-1 cells, BA $(10 \mu \mathrm{M})$ markedly upregulated the $i N O S$ transcription, increased the $i N O S / A r g-1$ ratio, and suppressed influenza $M$ gene replication at $24 \mathrm{~h}$ postinfection (Figure 2C). A lower dose of BA $(5 \mu \mathrm{M})$ also showed similar effects in primary peritoneal macrophages (Figure 2D).

We further evaluated the effects of BA $(5 \mu \mathrm{M})$ on the IFN signaling pathway in ANA-1 cells at 24 and $48 \mathrm{~h}$ postinfection. Influenza virus replicated in ANA-1 cells in a time-dependent manner, which was inhibited by BA treatment. We further compared $I F N-\beta, I L-10$, and interferon regulatory factor 3/7 (IRF-3/7) between untreated infected and BA-treated infected cells. The results indicate that BA treatment upregulates IFN- $\beta$ and IRF-3 transcription and downregulates transcription of $I L-10$ as shown in Figure 2E.

These results suggest that BA exerts its suppression of viral replication at an early stage by triggering macrophage M1 polarization and activating IFN signaling.

\section{BA Promotes IL-1 $\beta$ Cleavage and Cell-Cell Adhesion}

The IL-1 cytokine family cytokines are cytosolic proteins that exhibit inflammatory activity upon release into the extracellular space. The mature, cleaved form of IL- $1 \beta$ is closely related to the hyperactivated macrophage (Fabozzi et al., 2018).

We assessed the activation of IL-1 $\beta$ in ANA-1 cells at $24 \mathrm{~h}$ postinfection by immunofluorescence. As shown in Figure 3A, we observed that $1 \mu \mathrm{M}$ BA could increase levels of cleaved IL-1 $\beta$. In infected cells, BA significantly promoted virus-induced IL-1 $\beta$ cleavage (Figure 3B). Meanwhile, BA also significantly enhanced cell-cell adhesion to form macrophage aggregates.

To explore the influence of BA on macrophage migration, we used wild-type and $\mathrm{GFP}^{+}$ANA-1 macrophages and human BEAS-2B cells (Cardani et al., 2017) in the Transwell experimental system $\left(8.0 \mu \mathrm{m}\right.$ membrane). By tracking $\mathrm{GFP}^{+}$ ANA-1 cell migration, we observed that $\mathrm{H} 1 \mathrm{~N} 1$ infection resulted in an enhanced $\mathrm{GFP}^{+}$ANA-1 cell recruitment to infected BEAS$2 \mathrm{~B}$ and macrophages (Figure 3C). BA treatment improved $\mathrm{GFP}^{+}$ cell aggregation, and we detected more damaged cells (abnormal morphology) in infected BEAS-2B and ANA-1 cells.

To evaluate the role of TNF- $\alpha$ in BA-treated macrophages, we then used Etanercept, which is a commercially available soluble TNFR fusion protein inhibiting TNF- $\alpha$ at $70 \mu \mathrm{g} / \mathrm{mL}$. TNF- $\alpha$ inhibition appreciably abolished IL-1 $\beta$ cleavage and cell-cell adhesion triggered by BA (Figure 3D).

This evidence suggested that BA potentiated the hyperactivation and cell-cell adhesion of infected macrophages, and our results indicate that IL- $1 \beta$ and TNF- $\alpha$ possibly played a crucial role in this process.

\section{BA Triggers Discriminative Cellular Metabolic Responses}

The metabolic response plays a central role in linking cell responses in influenza virus-infected cells. Here, we used GCMS-based metabolomics to analyze the effects of BA treatment on the metabolic profile of ANA-1 cells.
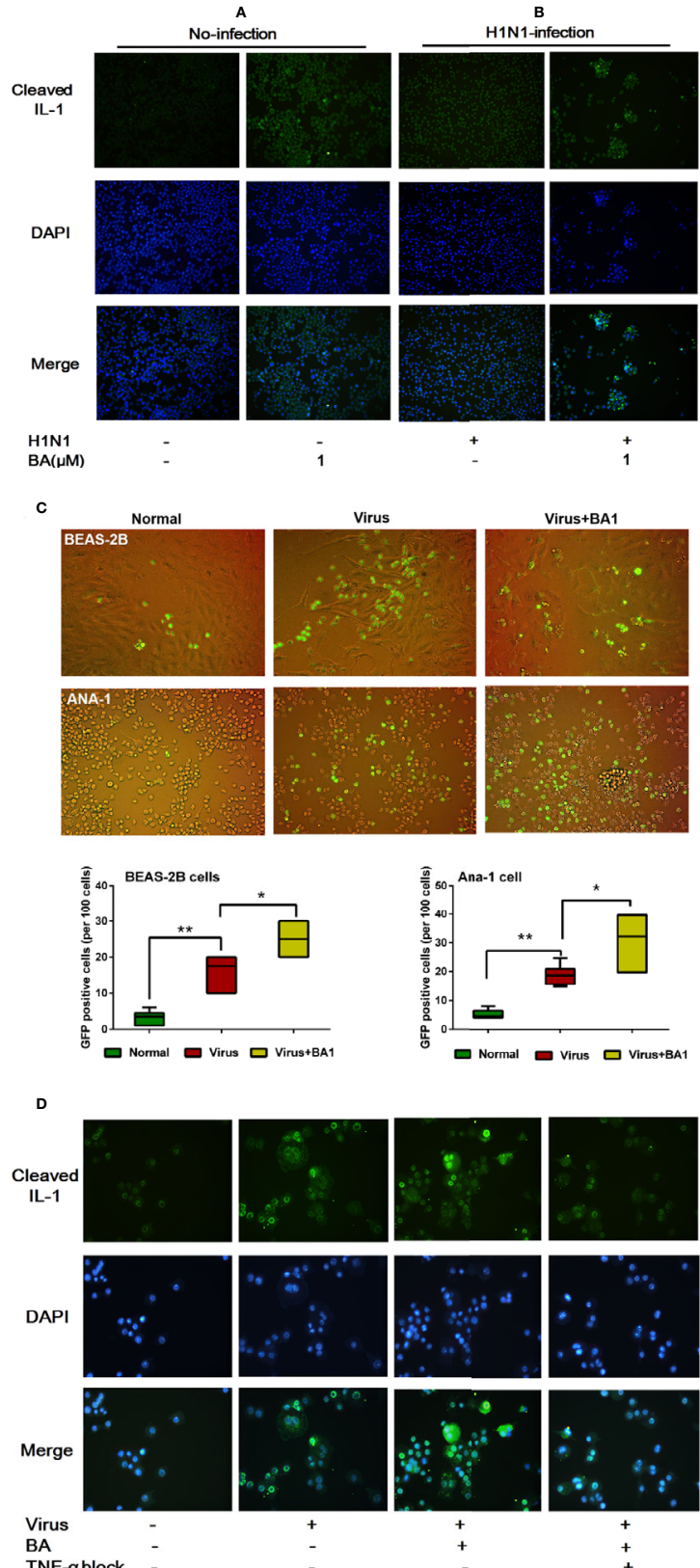

FIGURE 3 | BA amplifies influenza-induced macrophage IL-1 $\beta$ cleavage and cell-cell adhesion. Cells were infected with influenza virus H1N1 PR/8/34 strain at 10xTCID50. The medium was replaced after $1.5 \mathrm{~h}$ incubation, and then BA dilutions were added to 6 -well plates. Uninfected cells were used as the standard control. (A, B) At 24 h postinfection, ANA-1 cells were stained with a fluorescently labeled antibody against cleaved IL-1 1 . The cell nucleus was counterstained with DAPI. (C) In the Transwell system, GFP-labeled ANA-1 cells were inoculated in the upper chambers; the bronchial epithelial cells (BEAS-2B), and macrophage cells (ANA-1) in the lower chamber were infected and treated with BA. Macrophage recruitment and cell-cell adhesion were imaged using a Leica EL6000 microscope (Leica Microsystems CMS $\mathrm{GmbH}$ ). (D) Infected ANA-1 cells were treated with BA and Etanercept (TNF$\alpha$ inhibitor). IL-1 $\beta$ cleavage and cell-cell adhesion were observed under a Leica EL6000 microscope (Leica Microsystems CMS GmbH). Data are presented as mean $\pm \mathrm{SD}$. ${ }^{\star} \mathrm{p} \leq 0.05,{ }^{\star \star} \mathrm{p} \leq 0.01$ compared with virus samples. 
Cell samples were harvested for metabolomics analysis at $48 \mathrm{~h}$ postinfection. We obtained 1977 metabolite markers from GCMS by peak selection and alignment against the NIST library (only peaks with more than $70 \%$ similarity were considered as "detected"). Partial least squares discriminant analysis (PLS-DA) was undertaken to identify the differential metabolites of different groups (N: normal cells, V: virus-infected cells, BA: infected cells treated with $\mathrm{BA})$. BA treatment resulted in a noticeable deviation of metabolic responses in infected ANA-1 cells compared with normal and virus-infected samples (Figure 4A).

We identified 25 endogenous metabolites between normal and virus control samples, 17 endogenous metabolites between virus and BA samples, and explored the overall deviation in metabolism via a metabolite heat map. Compared to normal samples, the level of most metabolites in the virus-infected samples visibly increased, indicating that viral infection accelerated most metabolic reactions, and BA triggered a deviation of metabolites in infected samples (Figure 4B).

We identified 16 endogenous metabolites, which were significantly different between normal, virus control, and BAtreated cells. These metabolites covered, among others, organic acids, carbohydrates, amino acids, and lipids, which indicated that virus infection disturbed the overall metabolism. We, therefore, grouped metabolites as organic acids, amino acids, polyamines/ carbohydrates, and lipids/others as shown in Figure 4C. In the group of organic acids, BA reversed the upregulated levels of acetic acid, phosphate, and itaconic acid in infected samples. Apart from this, BA accelerated the degradation of amino acids ( $\beta$-alanine, $\mathrm{L}$ isoleucine) and lipids/others (methyl phosphate, 2,3-dihydroxy propyl phosphate, cholesta-5,24-diene) in infected samples.

Thus, BA resulted in a complicated metabolic response in ANA-1 cells at $48 \mathrm{~h}$ postinfection, which was possibly linked to its immune-stimulating effects on macrophages.

\section{DISCUSSION AND CONCLUSION}

Using both in vitro and in vivo models, we demonstrate in this study that BA treatment could ameliorate $\mathrm{H} 1 \mathrm{~N} 1 \mathrm{~A}$ virus-induced mortality via promotion of macrophage M1 polarization and IFN signaling pathway activation in the early stages of infection.

Macrophages are critical modulators of influenza A virus disease severity and the subsequent development of lethal pulmonary injury (Arora et al., 2018). Their shift between the classically activated (M1, proinflammatory) and alternatively activated (M2, anti-inflammatory) phenotypes has been recognized as a crucial factor in the initiation, progression, and termination of numerous inflammatory diseases (Zhang et al., 2018) and especially in influenza virus infection (Halstead et al., 2018). Modulation of macrophage polarization has previously been shown to inhibit or promote influenza infection by applying GMCSF (Sun et al., 2018) or Afatoxin B1 (Han et al., 2019), respectively. In this study, we confirm that the high mortality of infected mice is correlated with an abundant recruitment of macrophages to the lung and a high proportion of M1-polarized macrophages. BA treatment alleviated body weight loss and death of infected mice, and significantly reduced the recruitment of macrophages to the lung as measured by flow cytometry on the BALF. Meanwhile, we observed that BA treatment resulted in a maintained high proportion of M1-polarized macrophages. To elucidate the underlying molecular mechanisms of BA action, we next performed several in vitro experiments.

In murine ANA-1 and primary peritoneal macrophage cells, we further verified the M1 activation by BA treatment in multiple experiments (flow cytometry for CD86/CD206 and iNOS, ELISA for TNF- $\alpha$, and qPCR to determine the iNOS/Arg-1 ratio). This M1 polarization by BA after infection echoed similar M1promoting effects of $\mathrm{BA}$ in the tumor microenvironment ( $\mathrm{Li}$ and Wang, 2019). At the same time, we found that BA treatment promoted the upregulation of IFN- $\beta$ and IRF3 transcripts in ANA-1 cells, which is in line with other findings demonstrating IFN signaling pathway activation by BA treatment (Chu et al., 2015; Di Paolo and Shayakhmetov, 2016). Thus, macrophage M1 polarization and IFN signaling activation might be a key to the antiviral effects of BA.

In this study, we also evaluated macrophage M1 polarization by assessing IL-1 cleavage, which corresponds to macrophage hyperactivation (Fabozzi et al., 2018) and is involved in numerous inflammatory diseases (Ilyas et al., 2016). Through cellular immunofluorescence, we found that BA remarkably amplified the cleavage of IL-1 $\beta$ and cell-cell adhesion of the infected cells, which further verified macrophage M1 polarization. In our previous work, we demonstrated that BA treatment resulted in inhibitory effects on autophagosome accumulation in infected macrophages. Macrophage autophagy could limit acute toxic liver injury in mice through downregulation of IL-1 $\beta$ (Wang A. et al., 2016). Autophagy might be involved in the promotion of macrophage M1 polarization by BA, but the underlying mechanisms need to be further clarified in future experiments.

Viral infection triggers a cellular metabolic reprogramming, which is linked to immunity (Chen et al., 2014; Smallwood et al., 2017). In our previous work, we successfully used metabolomics to evaluate the systematic influence of a multicomponent natural product preparation on influenza virus infection (Sithisarn et al., 2013). Here, we also used GC-MS-based metabolomics to explore the impact of baicalin on macrophages in influenza virus infection. The metabolomic data presented in metabolic heat maps and PLSDA showed that BA induced significant differentiation of metabolites, deviating from normal and virus-infected samples. BA treatment mainly promoted a reduction of amino acids $(\beta$ alanine, L-isoleucine) and lipids/others (methyl phosphate, 2,3dihydroxy propyl phosphate, cholesta-5,24-diene), consistent with BA promoting an enhanced macrophage M1 polarization. The metabolomics data in this study provide a systematic evaluation; we aim to further clarify how metabolism and specific metabolites link to macrophage M1 polarization in further experiments.

The antiviral and anti-inflammatory effects of flavonoids have been reported in many studies (Zhi et al., 2019; Kiruthiga et al., 2020), but the underlying mechanism of these properties remains unclear. Regulation of macrophage recruitment and polarization is a promising field of research, and it may be beneficial to promote the research and development of flavonoids for antiviral and anti-inflammatory therapeutic applications. 


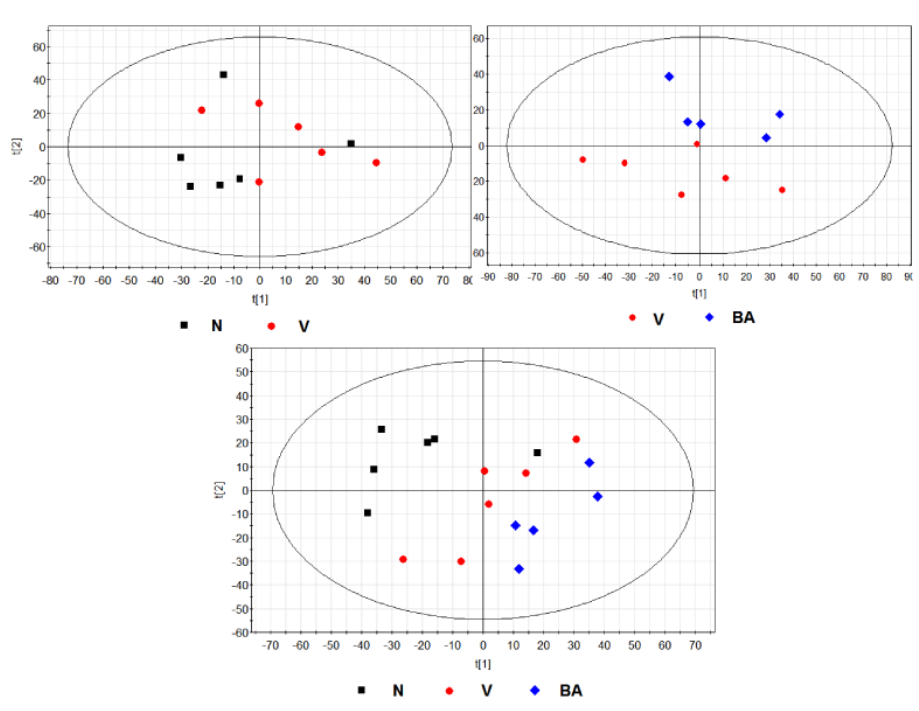

\begin{tabular}{|c|c|c|c|}
\hline & Normal & Virus & BA \\
\hline Acetic acid & & & \\
\hline phosphate & & & \\
\hline Glycine & & & \\
\hline Itaconic acid & & & \\
\hline B-Amino isobutyric acid & & & \\
\hline Lactic acid & & & \\
\hline Cholesterol & & & \\
\hline Cadaverine & & & \\
\hline Putrescine & & & \\
\hline Methyl-phosphate & & & \\
\hline P-Alamine & & & \\
\hline L-1soleucine & & & \\
\hline 2,3-dihydroxy-propy-phosphata & & & \\
\hline Glucose & & & \\
\hline Myo-Inositol & & & \\
\hline 3-hydroxy-cholesta-5,24-diene & & & \\
\hline $\begin{array}{l}\text { Metabolics Heatmap } \\
\text { ginal value-Mean) / Standard Deviation }\end{array}$ & -1.5 & 0 & x. \\
\hline
\end{tabular}

C Organic Acids
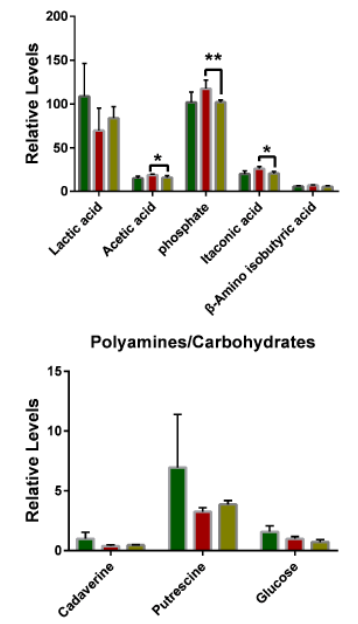

Amino Acids
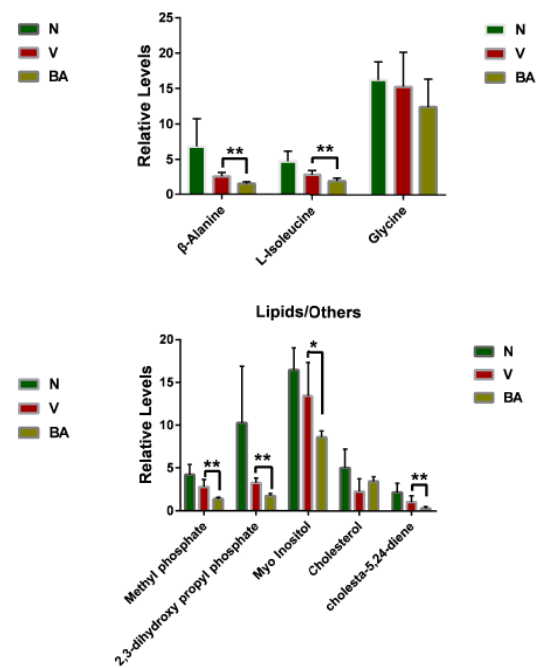

$=\begin{array}{lll}N \\ \text { va }\end{array}$

FIGURE 4 | Metabolomics analysis. At $48 \mathrm{~h}$ postinfection, cell samples were harvested for metabolomic analysis. (A) Partial least squares discriminant analysis (PLS-DA) was used to identify the obtained 1977 metabolite markers from GC-MS (N: healthy cells; V: virus-infected cells; BA: the infected cells treated by BA). (B) Metabolic heat map. For each metabolite, data was normalized using the $z$-score ((original valuemean) standard deviation) to values between -2 (dark blue), 0 (median; white), and 2 (maximum, dark red), to make a metabolite heat map. (C) Comparative analysis of major metabolites. The 16 metabolites were grouped as organic acids, amino acids, polyamines/carbohydrates, lipids/others. Data are presented as mean \pm SD. ${ }^{*} p \leq 0.05,{ }^{\star *} p \leq 0.01$ compared with virus samples. 


\section{DATA AVAILABILITY STATEMENT}

The datasets presented in this study can be found in online repositories. The names of the repository/repositories and accession number(s) can be found in the article/supplementary material.

\section{ETHICS STATEMENT}

The animal study was reviewed and approved by The Animal Experiment Committee of Fudan University (Shanghai, China).

\section{AUTHOR CONTRIBUTIONS}

PG and $\mathrm{HZ}$ carried out all in vitro and in vivo experiments in this study. WZ and HH carried out the immunoassays. CS, MC and CX performed the data analysis. $\mathrm{CH}$ participated in the design of the study. YC and XS conceived the study, participated in its design and coordination and helped to draft the manuscript. All authors contributed to the article and approved the submitted version.

\section{REFERENCES}

Arora, S., Dev, K., Agarwal, B., Das, P., and Syed, M. A. (2018). Macrophages: their role, activation, and polarization in pulmonary diseases. Immunobiology 223, 383-396. doi: 10.1016/j.imbio.2017.11.001

Cardani, A., Boulton, A., Kim, T. S., and Braciale, T. J. (2017). Alveolar macrophages prevent lethal influenza pneumonia by inhibiting infection of type-1 alveolar epithelial cells. PloS Pathog. 13, e1006140. doi: 10.1371/ journal.ppat.1006140

Chandler, J. D., Hu, X., Ko, E. J., Park, S., Lee, Y. T., Orr, M., et al. (2016). Metabolic pathways of lung inflammation revealed by high-resolution metabolomics (HRM) of H1N1 influenza virus infection in mice. Am. J. Physiol. Regul. Integr. Comp. Physiol. 311, R906-R916. doi: 10.1152/ajpregu.00298.2016

Chen, L., Fan, J., Li, Y., Shi, X., Ju, D., Yan, Q., et al. (2014). Modified Jiu Wei Qiang Huo decoction improves dysfunctional metabolomics in influenza A pneumoniainfected mice. BioMed. Chromatogr. 28, 468-474. doi: 10.1002/bmc.3055

Chu, M., Xu, L., Zhang, M. B., Chu, Z. Y., and Wang, Y. D. (2015). Role of baicalin in anti-influenza Virus A as a potent inducer of IFN-gamma. Biomed. Res. Int. 2015, 263630. doi: 10.1155/2015/263630

da Silva, L. C., da Silva, M. V., and Correia, M. T. (2017). Editorial: New frontiers in the search of antimicrobials agents from natural products. Front. Microbiol. 8, 210. doi: $10.3389 /$ fmicb. 2017.00210

Di Paolo, N. C., and Shayakhmetov, D. M. (2016). Interleukin $1 \alpha$ and the inflammatory process. Nat. Immunol. 17, 906-913. doi: 10.1038/ni.3503

Ding, Y., Dou, J., Teng, Z., Yu, J., Wang, T., Lu, N., et al. (2014). Anti-viral activity of baicalin against influenza A $(\mathrm{H} 1 \mathrm{~N} 1 / \mathrm{H} 3 \mathrm{~N} 2)$ virus in cell culture and in mice and its inhibition of neuraminidase. Arch. Virol. 159, 3269-3278. doi: 10.1007/ s00705-014-2192-2

Evavold, C. L., Ruan, J., Tan, Y., Xia, S., Wu, H., and Kagan, J. C. (2018). The poreforming protein gasdermin D regulates interleukin-1 secretion from living macrophages. Immunity 48, 35-44.e6. doi: 10.1016/j.immuni.2017.11.013

Fabozzi, G., Oler, A. J., Liu, P., Chen, Y., Mindaye, S., Dolan, M. A., et al. (2018). Strand-specific dual rna sequencing of bronchial epithelial cells infected with influenza A/H3N2 viruses reveals splicing of gene segment 6 and novel hostvirus interactions. J. Virol. 92, e00518-e00518. doi: 10.1128/JVI.00518-18

Halstead, E. S., Umstead, T. M., Davies, M. L., Kawasawa, Y. I., Silveyra, P., Howyrlak, J., et al. (2018). GM-CSF overexpression after influenza a virus infection prevents mortality and moderates M1-like airway monocyte/ macrophage polarization. Respir. Res. 19, 3. doi: 10.1186/s12931-017-0708-5

Han, S., Wang, W., Wang, S., Wang, S., Ju, R., Pan, Z., et al. (2019). Multifunctional biomimetic nanoparticles loading baicalin for polarizing

\section{FUNDING}

This work was supported by the Key Program of Nation Nature Science Foundation of China (No.U1604283), Shanghai Science and Technology Funds (Nos. 17ZR1401700), and Shanghai Municipal Health Commission project (Nos.201740200).

\section{ACKNOWLEDGMENTS}

All authors would like to show our deepest gratitude to Shanghai Sensichip Infotech Co., Ltd. and Dongguan Jinmeiji Pharmaceutical Co., Ltd. for their technical support.

\section{SUPPLEMENTARY MATERIAL}

The Supplementary Material for this article can be found online at: https://www.frontiersin.org/articles/10.3389/fphar.2020. 01298/full\#supplementary-material

tumor-associated macrophages. Nanoscale 11, 20206-20220. doi: 10.1039/ c9nr03353j

http://www.who.int/influenza/surveillance_monitoring/updates/latest_update_ GIP_surveillance/en/.

Ilyas, G., Zhao, E., Liu, K., Lin, Y., Tesfa, L., Tanaka, K. E., et al. (2016). Macrophage autophagy limits acute toxic liver injury in mice through down regulation of interleukin-1 $\beta$. J. Hepatol. 64, 118-127. doi: 10.1016/j.jhep.2015.08.019

Kiruthiga, C., Devi, K. P., Nabavi, S. M., and Bishayee, A. (2020). Autophagy: a potential therapeutic target of polyphenols in hepatocellular carcinoma. Cancers (Basel) 12, 562. doi: 10.3390/cancers 12030562

Li, R., and Wang, L. (2019). Baicalin inhibits influenza virus A replication via activation of type I IFN signaling by reducing miR-146a. Mol. Med. Rep. 20, 5041-5049. doi: 10.3892/mmr.2019.10743

Li, C., Xu, M. M., Wang, K., Adler, A. J., Vella, A. T., and Zhou, B. (2018). Macrophage polarization and meta-inflammation. Transl. Res. 191, 29-44. doi: $10.1016 /$ j.trsl.2017.10.004

Lin, S., Liu, N., Yang, Z., Song, W., Wang, P., Chen, H., et al. (2010). GC/MS-based metabolomics reveals fatty acid biosynthesis and cholesterol metabolism in cell lines infected with influenza A virus. Talanta 83, 262-268. doi: 10.1016/ j.talanta.2010.09.019

Murray, P. J., Allen, J. E., Biswas, S. K., Fisher, E. A., Gilroy, D. W., Goerdt, S., et al. (2014). Macrophage activation and polarization: nomenclature and experimental guidelines. Immunity 41, 14-20. doi: 10.1016/j.immuni.2014. 06.008

Nayak, M. K., Agrawal, A. S., Bose, S., Naskar, S., Bhowmick, R., Chakrabarti, S., et al. (2014). Anti-viral activity of baicalin against influenza virus H1N1pdm09 is due to modulation of NS1-mediated cellular innate immune responses. J. Antimicrob. Chemother. 5, 1298-1231. doi: 10.1093/jac/dkt534

Rello, J. (2017). Theranostics in severe influenza. Lancet Respir. Med. 5, 91-92. doi: 10.1016/S2213-2600(17)30005-X

Ridnour, L. A., Cheng, R. Y., Weiss, J. M., Kaur, S., Soto-Pantoja, D. R., Basudhar, D., et al. (2015). NOS inhibition modulates immune polarization and improves radiation-induced tumor growth delay. Cancer Res. 75, 2788-2799. doi: 10.1158/ 0008-5472.CAN-14-3011

Schmittgen, T. D., and Livak, K. J. (2008). Analyzing real-time PCR data by the comparative C(T) method. Nat. Protoc. 3, 1101-1108. doi: 10.1038/ nprot. 2008.73

Sithisarn, P., Michaelis, M., Schubert-Zsilavecz, M., and Cinatl, J. Jr. (2013). Differential antiviral and anti-inflammatory mechanisms of the flavonoids biochanin A and baicalein in $\mathrm{H} 5 \mathrm{~N} 1$ influenza A virus-infected cells. Antiviral Res. 97, 41-48. doi: 10.1016/j.antiviral.2012.10.004 
Smallwood, H. S., Duan, S., Morfouace, M., Rezinciuc, S., Shulkin, B. L., Shelat, A., et al. (2017). Targeting metabolic reprogramming by influenza infection for therapeutic intervention. Cell Rep. 19, 1640-1653. doi: 10.1016/j.celrep.2017.04.039

Sun, Y., Liu, Z., Liu, D., Chen, J., Gan, F., and Huang, K. (2018). Low-level aflatoxin b1 promotes influenza infection and modulates a switch in macrophage polarization from M1 to M2. Cell Physiol. Biochem. 49, 11101126. doi: $10.1159 / 000493294$

Tisoncik-Go, J., Gasper, D. J., Kyle, J. E., Eisfeld, A. J., Selinger, C., Hatta, M., et al. (2016). integrated omics analysis of pathogenic host responses during pandemic H1N1 influenza virus infection: the crucial role of lipid metabolism. Cell Host Microbe 19, 254-266. doi: 10.1016/j.chom.2016.01.002

Vergadi, E., Ieronymaki, E., Lyroni, K., Vaporidi, K., and Tsatsanis, C. (2017). Akt signaling pathway in macrophage activation and M1/M2 polarization. J. Immunol. 198, 1006-1014. doi: 10.4049/jimmunol.1601515

Wang, X., Zhu, P., Pi, F., Jiang, H., Shao, J., Zhang, Y., et al. (2016). A sensitive and simple macrophage-based electrochemical biosensor for evaluating lipopolysaccharide cytotoxicity of pathogenic bacteria. Biosens. Bioelectron. 81, 349-357. doi: 10.1016/j.bios.2016.03.007

Wang, A., Huen, S. C., Luan, H. H., Yu, S., Zhang, C., Gallezot, J. D., et al. (2016). Opposing effects of fasting metabolism on tissue tolerance in bacterial and viral inflammation. Cell 166, 1512-1525.e12. doi: 10.1016/j.cell.2016.07.026

Weinhofer, I., Zierfuss, B., Hametner, S., Wagner, M., Popitsch, N., Machacek, C., et al. (2018). Impaired plasticity of macrophages in X-linked adrenoleukodystrophy. Brain 141, 2329-2342. doi: 10.1093/brain/awy127

Zhang, N., Bao, Y. J., Tong, A. H., Zuyderduyn, S., Bader, G. D., Malik Peiris, J. S., et al. (2018). Whole transcriptome analysis reveals differential gene expression profile reflecting macrophage polarization in response to influenza A H5N1 virus infection. BMC Med. Genomics 11, 20. doi: 10.1186/s12920-018-0335-0

Zhi, H. J., Zhu, H. Y., Zhang, Y. Y., Lu, Y., Li, H., and Chen, D. F. (2019). In vivo effect of quantified flavonoids-enriched extract of Scutellaria baicalensis root on acute lung injury induced by influenza A virus. Phytomedicine 57, 105-116. doi: 10.1016/j.phymed.2018.12.009

Zhou, L., Ren, R., Yang, L., Bao, C., Wu, J., Wang, D., et al. (2017). Sudden increase in human infection with avian influenza A (H7N9) virus in China, SeptemberDecember 2016. Western Pac. Surveill. Response J. 8, 6-14. doi: 10.5365/ WPSAR.2017.8.1.001

Zhu, H. Y., Han, L., Shi, X. L., Wang, B. L., Huang, H., Wang, X., et al. (2015). Baicalin inhibits autophagy induced by influenza A virus H3N2. Antiviral. Res. 113, 62-70. doi: 10.1016/j.antiviral.2014.11.003

Conflict of Interest: The authors declare that the research was conducted in the absence of any commercial or financial relationships that could be construed as a potential conflict of interest.

Copyright (C) 2020 Geng, Zhu, Zhou, Su, Chen, Huang, Xia, Huang, Cao and Shi. This is an open-access article distributed under the terms of the Creative Commons Attribution License (CC BY). The use, distribution or reproduction in other forums is permitted, provided the original author(s) and the copyright owner(s) are credited and that the original publication in this journal is cited, in accordance with accepted academic practice. No use, distribution or reproduction is permitted which does not comply with these terms. 